\title{
Virtual Performance Evaluation of Automotive Radar Concepts in Realistic Traffic Environments
}

\author{
$\begin{array}{llll}\text { C. Sturm } & 1 & \text { S. Knörzer } & \\ & \text { T. Zwick } & \text { W. Wiesbeck }\end{array}$
}

\begin{abstract}
In this paper a simulation approach is presented, that allows for virtually investigating the performance of automotive radar systems regarding different system parameters. This is achieved by combining dedicated simulation models for the radar transmitter and receiver with a deterministic propagation simulator based on ray tracing. This approach is in particular suitable for investigating the capabilities of beamforming algorithms in radar systems with multiple antenna receivers. The configuration of the developed tool is explained in detail and the performance of different array configurations for classic digital beamforming and the MUSIC algorithm is evaluated.
\end{abstract}

\section{INTRODUCTION}

In recent years the application of radar sensors in luxury class cars has become very popular in order to realize adaptive cruise control systems that automatically adapt to the speed of the vehicle ahead. In order to allow for a distinction of the vehicles on the different lanes, these systems have to provide two-dimensional radar sensing that detects the position in both distance and azimuth angle. This can be accomplished by employing multiple antenna processing techniques, which exploit the phase differences between the signals received at different spatial positions in order to determine the directions of arrival of the received signals. For that purpose numerous algorithms exist, that are based on different mathematical approaches.

In the presented work a simulation tool that allows for the investigation of the performance of these multiple antenna processing techniques in realistic street scenarios has been developed. Moreover, in this tool the simulations are performed with realistic radar signals, in order to completely model the entire signal chain and evaluate typical system aspects like e.g. the influence of the signal to noise ratio (SNR) on the quality of the radar image. This has been achieved by developing a suitable interconnection between a radar signal and multiple antenna processing simulator and a dedicated deterministic wave propagation model.

\section{SIMULATION MODEL}

The developed simulation tool combines simulation components for a radar transmitter and receiver, which are implemented in MatLab, with a deterministic propagation simulator based on raytracing implemented in $\mathrm{C}++$. The different components are now regarded in detail.

\subsection{Transmitter and Transmitted Waveform}

\begin{tabular}{|c|c|}
\hline Parameter & Value \\
\hline PN code & M-sequence \\
\hline Modulation & BPSK \\
\hline Chip rate & $\begin{array}{c}\text { Root raised cosine } \\
\text { roll-off }(\mathrm{r}=1)\end{array}$ \\
\hline Transmit filter & $\approx 96 \mathrm{MHz}$ \\
\hline $\begin{array}{c}\text { Transmit signal } \\
\text { bandwidth }\end{array}$ & $\begin{array}{c}32767 \mathrm{Chips} \\
(683 \mu \mathrm{s})\end{array}$ \\
\hline Transmit frame length & $24 \mathrm{GHz}$ \\
\hline Carrier frequency & \\
\hline
\end{tabular}

Table 1: System parameters of the pseudo noise radar transmitter.

The transmitter simulation component generates a phase coded pseudo-noise (PN) transmit signal. This choice has been made since it allows for the transmission of data in parallel [1]. The PN signal is derived from an M-sequence by binary phase shift keying (BPSK). The resulting transmit signal in the baseband is described by Eq. 1 .

$$
\left.x(t)=\sum_{i=-\infty}^{\infty} a(i) g(t-i T)\right)
$$

with $T$ denoting the chip duration, $g(t)$ being the impulse response of the transmit filter, and $a(i)$ representing the phase coded chips. The PN sequence has a length of $32767\left(2^{15}-1\right)$ and is transmitted with a chip rate of $48 \mathrm{MChip} / \mathrm{s}$, which results in a

\footnotetext{
${ }^{1}$ Institut für Hochfrequenztechnik und Elektronik (IHE), Universität Karlsruhe (TH), Kaiserstr. 12, 76131 Karlsruhe, Germany, e-mail: christian.sturmeihe.uka.de, tel.: +49 721608 7675, fax: +49721691865.

${ }^{2}$ Institut für Hochfrequenztechnik und Elektronik (IHE), Universität Karlsruhe (TH), Kaiserstr. 12, 76131 Karlsruhe, Germany, e-mail: sandra. knoerzer@i he . uka. de, tel.: +49 721608 2525, fax: +49 721691865.

${ }^{3}$ Institut für Hochfrequenztechnik und Elektronik (IHE), Universität Karlsruhe (TH), Kaiserstr. 12, 76131 Karlsruhe, Germany, e-mail: thomas. zwick@ihe. uka. de, tel.: +49 721608 2522, fax: +49 721691865.

${ }^{4}$ Institut für Hochfrequenztechnik und Elektronik (IHE), Universität Karlsruhe (TH), Kaiserstr. 12, 76131 Karlsruhe, Germany, e-mail: werner.wiesbeck@kit.edu, tel.: +49 721608 3303, fax: +49 721691865 .
} 
total frame duration of $683 \mu \mathrm{s}$. With the application of a root raised cosine roll-off filter (roll-off factor 1) the analog output signal has a bandwidth of approx. $96 \mathrm{MHz}$. In the simulator the signals are oversampled in the roll-off filter in order to generate a quasicontinuous output signal. Finally the signal is converted to the carrier frequency of $24 \mathrm{GHz}$ in a bandpass subsampling representation. The most important system parameters are summarized in Table 1 .

\subsection{Traffic and Wave Propagation Model}

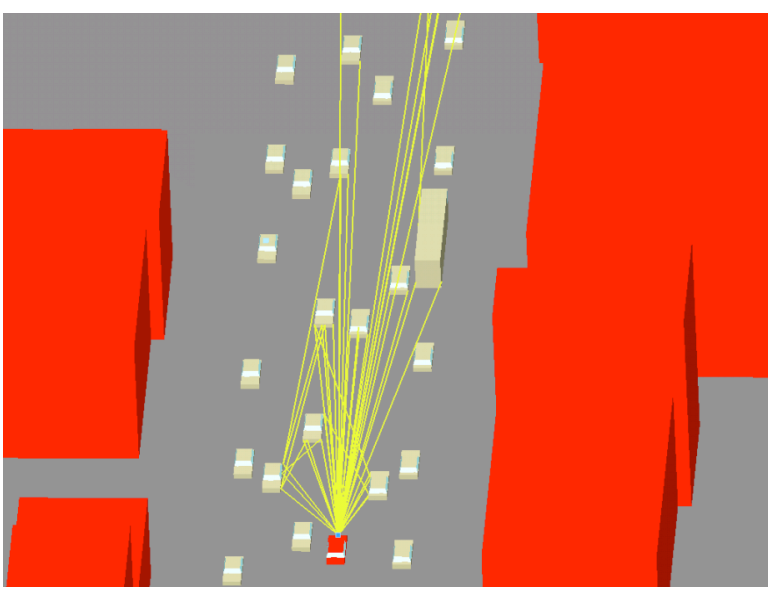

Figure 1: Investigated traffic scenario.

In order to adequately model the road scenario including the wave propagation and the scattering of the transmitted signal, a dedicated simulator is employed [2]. This software tool first creates a typical road environment from a stochastic traffic model. The result is a polygon object model of the scenario, in which each object (cars, trucks, buildings, etc.) is represented by a composition of several polygons. One car is equipped with the transmit and receive antennas of the quasi-monostatic radar system. The antennas can be assigned arbitrary gain patterns. In a second step with ray tracing algorithms all possible reflections of the transmit signal towards the position of the receiving antenna are determined. In order to consider the multiple antenna configuration at the receiver, the simulations are repeated for each receiving antenna position individually. The propagation path search also considers higher order reflections up to an order of 5 . In Fig. 1 the randomly created scenario that has been used for the investigations in this paper is shown. The car that is equipped with the radar system is colored in red. The yellow lines indicate the determined propagation paths from the transmit antenna to the receive antenna.

For every determined propagation path the occurring time shift, attenuation, and phase shift are individually calculated respecting the dielectric material properties of the reflecting object and the antenna gain for the relevant direction. Then, all propagation paths are coherently superimposed in order to obtain the instantaneous channel impulse response. The absolute value of the channel impulse resulting for the scenario in Fig. 1 is shown in Fig. 2.

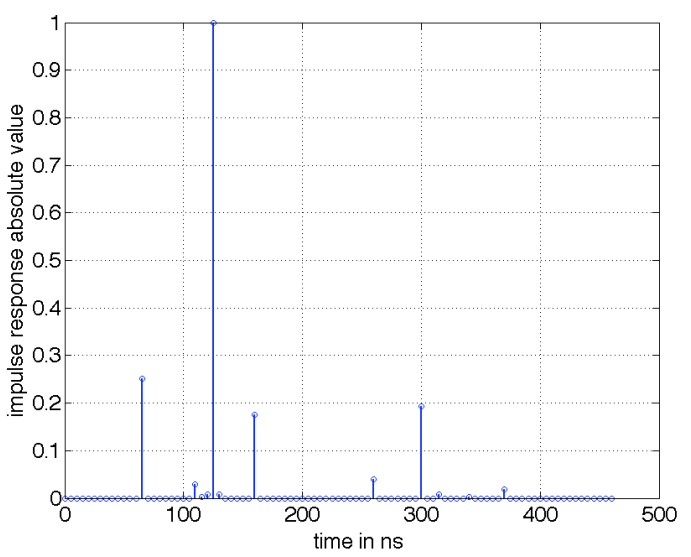

Figure 2: Impulse response obtained for the scenario shown in Fig 1.

The received signals $y_{\mathrm{i}}(t)$ are calculated by convoluting the transmit signal $x(t)$ with the channel impulse response for every receiving antenna. It must be noted that by this procedure the Doppler effect is neglected. However, it has been assured that the chosen frame length is sufficiently short and the phases of the received signal components do not significantly shift during the frame length duration.

\subsection{Multiple Antenna Radar Receiver}

The simulation model assumes an individual receiver for every receiving antenna. The calculation of the radar image consists of two steps. First, at every individual receiver a complex-valued radar range profile is computed. Second, the radar range profiles from all antennas are combined to a two-dimensional radar image by applying multiple antenna techniques. The different steps will now be regarded in detail.

In the receiver model, first the received signal $y_{\mathrm{i}}(t)$ at every receiver is converted back to the baseband. Then the cross-correlation function of each received signal and the transmitted signal is calculated in the baseband over one transmit frame length duration $T_{f}$, as described in Equation 2.

$$
R_{y x, i}(\tau)=\int_{0}^{T_{f}} y_{i}(t) x(t-\tau) d t
$$

The result of this cross-correlation function directly corresponds to the radar range profile.

In the subsequent multiple antenna processing the complex-valued range profiles from all receiving 
antennas are jointly processed. Assuming the receiver has an antenna array of $P$ elements, the individual cross-correlation functions $R_{y x, i}(\tau)$ obtained from the operation described in (2) can be arranged in the array signal vector

$$
\vec{R}_{y x}(\tau)=\left[\begin{array}{llll}
R_{y x, 1}(\tau) & R_{y x, 2}(\tau) & \ldots & R_{y x, P}(\tau)
\end{array}\right]^{\mathrm{T}}
$$

In the following, the application of two widely used multiple antenna processing approaches will be described and investigated. In order to limit the complexity of the equations, a horizontally oriented linear antenna array with constant element spacing $d$ will be regarded. As a common basis both approaches share the definition of a beam-steering vector, which is a vector that describes the samples of the complex wave front at the receiving antenna positions, provided that one single plane wave is impinging on the array from the azimuth angle $\psi$

$$
\vec{b}(\psi)=\left[\begin{array}{llll}
1 & e^{j \sin (\psi) 2 \pi d / \lambda} & \ldots & e^{j \sin (\psi) 2 \pi(P-1) d / \lambda}
\end{array}\right]^{\mathrm{T}}
$$

First, the classic Fourier transform based approach will be regarded, which consists in simply adding the elements of array signal vector with additional phase shifts. This corresponds to multiplying this vector with the beam-steering vector. The Radar image intensity $I$ for a given range cell $\tau$ and azimuth direction $\psi$ is obtained as

$$
I(\tau, \psi)=\left|\vec{R}_{y x}^{\mathrm{T}}(\tau) \cdot \vec{b}(\psi)\right|^{2}
$$

Second, a multiple antenna processing approach will be applied that is much more sophisticated and allows for considerably higher angular resolution. This approach is named MUSIC (Multiple Signal Classification) and has first been described in [3]. It operates on the eigen-structure of the correlation matrix of the array signal vector. In this algorithm the (pseudo) Radar image intensity is defined as

$$
I(\tau, \psi)=\frac{1}{\sum_{i=1}^{P-Q}\left|\vec{b}(\psi) \vec{e}_{n, i}\right|^{2}}
$$

with $\vec{e}_{n, i}$ being the eigenvectors that are related to the eigenvalues of the correlation matrix $\mathbf{R}_{R R}$ of the array signal vector defined in (3) with values equal to the spectral noise density $(P-Q$ is the number of these eigenvalues).

\section{SIMULATION RESULTS}

As an example here simulation results for two different array geometries with the application of both multiple antenna techniques that have been discussed are presented. The regarded array geometries consist of only four receiving antennas, which allows for cost efficient hardware architectures as typically required for automotive applications. The first array geometry that is regarded is the typical configuration with half-wavelength distance between the elements. A sketch of the geometry is shown in Fig. 3. The numbers at the antenna elements indicate the weighting coefficients of the individual elements (used in the Fourier transform based approach only).

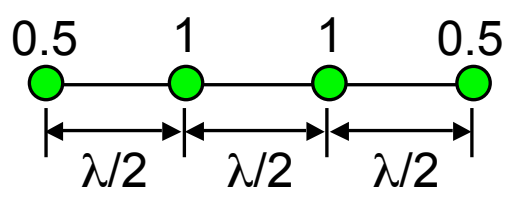

Figure 3: Investigated array geometry 1.

The radar image obtained for the traffic scenario in Fig. 1 with the classical Fourier transform based digital beamforming algorithm described in (5) is shown in Fig. 4.

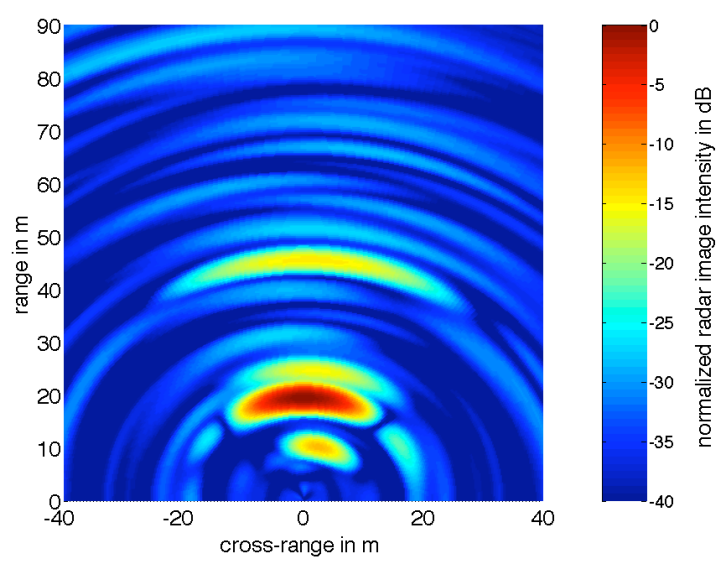

Figure 4: Radar image obtained with Fourier transform based beamforming for geometry 1 .

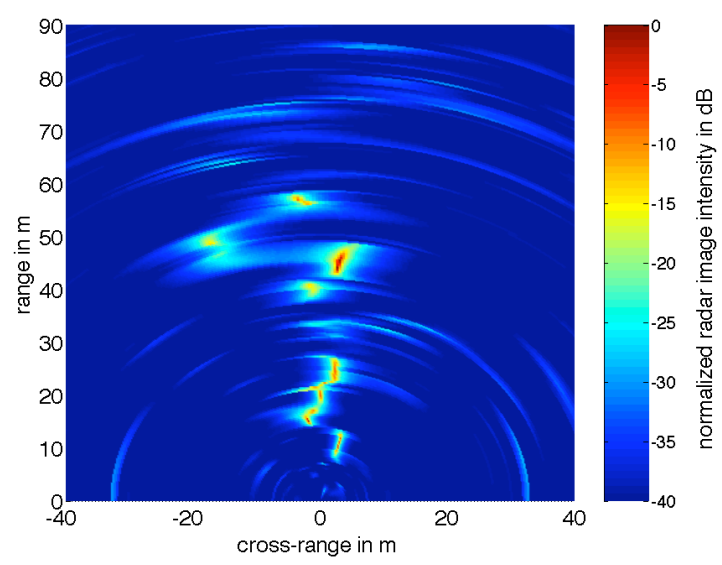

Figure 5: Radar image obtained with the MUSIC algorithm for geometry 1 .

Due to the low number of receiving antennas the angular resolution is very low. Only four reflecting objects can be detected in the radar image, the 
achievable position accuracy of these objects is rather low. Moreover, sidelobes caused by the strongest reflection are clearly visible. The radar image obtained for the same scenario with the MUSIC algorithm is shown in Fig. 5. With the same array configuration a very high angular resolution is achieved, at least seven reflecting objects can be clearly distinguished in the radar image. Moreover, sidelobes like in the Fourier based processing do not occur and the background noise is clearly lower.

The second investigated array geometry is shown in Fig. 6. The idea behind this geometry has been to use an irregular spacing with an increase of the total baselength of the array in order to achieve better angular resolution while at the same time keeping the half-wavelength spacing for two elements in order to avoid grating lobes. The numbers again indicate the weighting coefficients for the Fourier transform based approach.

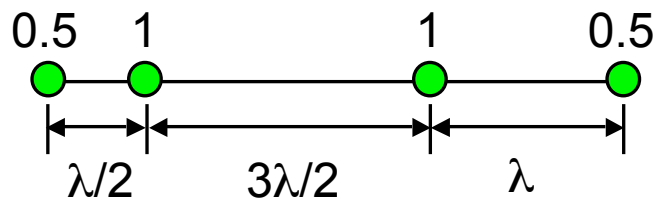

Figure 6: Investigated array geometry 2.

The radar image obtained with the Fourier transform based approach for this geometry is shown in Fig. 7 and the result with the MUSIC algorithm is shown in Fig. 8, respectively.

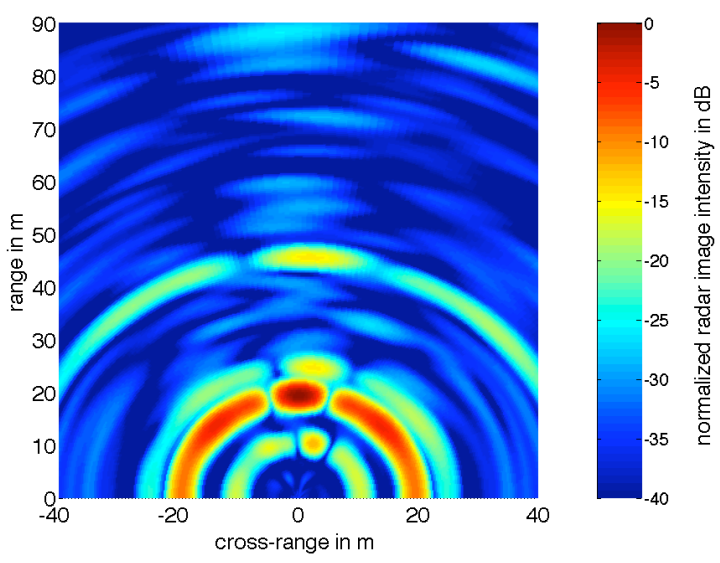

Figure 7: Radar image obtained with Fourier transform based beamforming for geometry 2 .

In Fig. 7 it can be observed that indeed the angular extension of the main maxima becomes smaller. However, even though there are no grating lobes, the sidelobe levels drastically increase. On the other hand from Fig. 8 it can be seen that for the MUSIC algorithm this problem does not appear. However, the imaging capability is worse and the background noise increases when using the array geometry 2 .

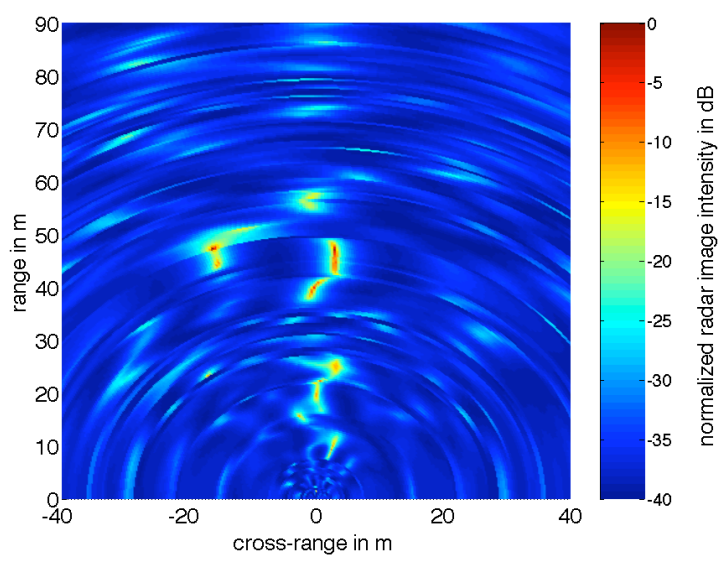

Figure 8: Radar image obtained with the MUSIC algorithm for geometry 2 .

\section{SUMMARY AND CONCLUSIONS}

In this paper a simulation tool has been presented, that allows for investigating the entire signal flow in an automotive radar application considering the wave propagation in a realistic street scenario. With this tool the performance of different system configurations can be virtually investigated without the need for building prototypes. As an example the performance of different multiple antenna processing techniques and different antenna array configurations has been presented. However, there are many additional application areas for the developed tool, like e.g. the performance investigation of different radar waveforms. Also the influence of noise and interference can be assessed under realistic conditions. Hence the developed software tool can be regarded a comprehensive solution for the virtual performance evaluation of automotive radar concepts.

\section{References}

[1] C. Sturm, S. Schulteis, and W. Wiesbeck, "Twodimensional radar imaging with scattered PSKmodulated communication signals," 4th European Radar Conference, pp. 134-137, Munich, Germany, Oct. 2007.

[2] J. Maurer, T. Fügen, S. Knörzer, and W. Wiesbeck, "A ray-optical approach to model the inter-vehicle transmission channel,“ Frequenz, journal of RF-engineering and telecommunications, vol. 60, no. 5-6, pp. 95-98, May/June 2006

[3] R. O. Schmidt. "Multiple Emitter Location and Signal Parameter Estimation", IEEE Transactions on Antennas and Propagation, vol. 34, pp. 276280, 1986. 